\title{
Auto Quality Testing and Filling Station by using NI LabVIEW and Specially Designed OFS
}

\author{
G.Venkateswarlu \\ Department of EIE, CVR College of Engineering, Hyderabad, India \\ Email: venkigummadilli@gmail.com
}

\begin{abstract}
Quality testing for various parameters of the final product of the miniature plant is performed by using various sensors. The sensors include LDR, PH meter, Thermo couple, Optical fiber sensor (OFS). pH, level, temperature, weight and turbidity are few parameters that are tested in this miniature industrial prototype. Miniature model of a bottle filling plant has been designed to implement various sensors and actuators. OFS is an optical sensor that converts weight into electrical signal. It is based on the micro bending phenomenon. All the sensor signals are taken and actuator signals are given by the LabVIEW Data Acquisition through Compact Field Point. The automation, data logging and process controlling has been achieved by a Virtual Instrument (VI) designed on National Instrument's LabVIEW platform.

Motivation for the project: Any automated industrial plant or process needs to be monitored by sensors and controlled by a controller. The controllers to handle such sophisticated industrial processes need to be inevitably flexible and easier to program. Modular control is also a desirable tradeoff. All these requirements are satisfied by the graphical programming of LabVIEW VI.Iterative uniformity being the most important factor of output of any process, quality of the end products is tested for uniformity of various parameters. This quality testing helps in giving the system a feedback and a control input. The tested values are monitored by data logging in the form of graphs and charts.
\end{abstract}

Index Terms: OFS, LabVIEW, CFP, turbidity, LDR.

\section{INTRODUCTION}

The literature study of this project begins with the study of various sensors used in industries and a comparison between them in terms of flexibility, performance, environmental tolerance and other features. Also, the most suitable platform to work upon, in order to create a flexible program that is easy to design and handle i.e., LabVIEW is studied. The specifications and installation of NI CFP 2020, AI 100, AIO 610 and other NI modules are studied.

The project uses a prototype model of a miniature bottle filling plant where general instrumentation practices are observed and implemented. Right from sensors applications to controlling and actuation are dealt in the project. This involves physical setup of a process plant, devices, components, transducers, sensors, actuators, DAQ, CFP etc (Fig.1). An empty bottle is placed in the start of the process over a continuously running conveyor belt. An LDR sensor detects the presence of the bottle and the conveyor stops for a programmed time delay. During this delay, the DC solenoid valve is actuated to be opened and the liquid is filled. After filling the conveyor resumes for the bottle to be processed further towards quality testing end. The fluid in the filling tank is monitored for temperature $\mathrm{pH}$ and level. The filled bottle is monitored for weight within standards. A DAQ and CFP manufactured by National Instruments are used for data acquisition from field points to the software code. National Instruments Compact field point is used to interface the hardware with the virtual instrument code developed in LabVIEW [9]. LabVIEW is a graphical programming tool that enables the user to develop programs swiftly without even worrying about syntax. Various functions have been used modularly in order to run each independent process in parallel. The data obtained from the process is also recorded and stored in various formats directly from the virtual instrument.

The paper is organized as follows .Section I describes introduction to literature study, quality testing filling and techniques used for measurement of physical variables considered and controlling in our process.

Section II: Block diagram of the project considered

Section III: OFS and hardware setup.

Section IV: LabVIEW interfacing and VI code

Section V: results

Section VI: Conclusion

\section{BLOCK DIAGRAM OF THE APPLICATION}

Industrial manufacturing has revolutionized with advances and developments in sensor technology and controller technology. Automation and process control has come into mainframe instrumentation as the follow up of industrial automation and control. Many tools have been developed as platforms for building automation programs. Customized microcontrollers, PLC, LabVIEW are few to name some. In any of these platforms, automation is achieved a network of information exchange if various parameters and a consequent instruction command embedded into the program code.

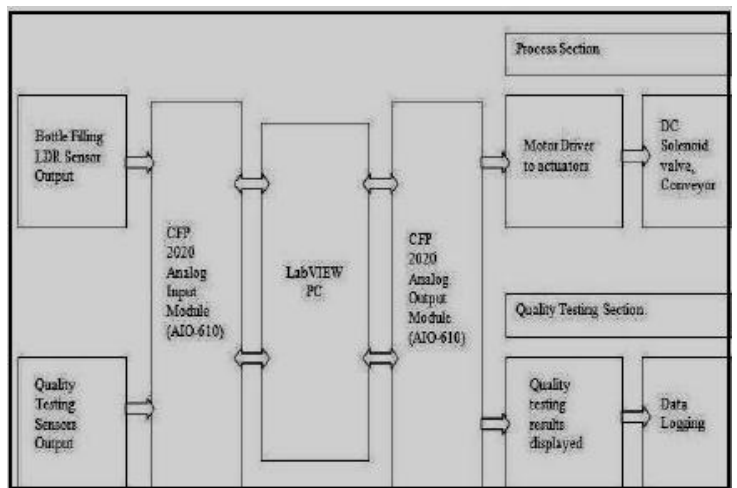

Figure 1.Block diagram of testing and filling station 
The project uses a prototype model of a bottle filling plant where general instrumentation practices are observed and implemented. Right from sensors applications to controlling and actuation are dealt in the project. This involves physical setup of a process plant, devices, components, transducers, sensors, actuators, DAQ, CFP etc.

Quality testing: In any industrial process various process parameters are involved and they need to be measured, compared, modified and controlled for the output to meet the desired set points with a reasonable tolerance. Thus the output or product of the process is tested for various parameters to check for quality. The main objective of quality testing is to check for uniformity and precision of the outcome. Thus various transducers and sensors are used at various stages of the process like raw material testing, intermediate processes monitoring and end product testing. This measurement is also given as feedback to the process for process correction and adjustment. All the sensor signals are data-logged and plotted as graphs and charts by the LabVIEW VI. pH, level, temperature and weight are few parameters that are tested in this miniature industrial prototype.

In this paper we are using Virtual Instrumentation for data acquisition and control.Virtual Instrumentation is the use of customizable software and modular measurement hardware to create user-defined measurement systems, called virtual instruments. LabVIEW (Laboratory Virtual Instrumentation Engineering Workbench) is one such tool [7]. It is a highly productive development environment that engineers and scientists use for graphical programming and unprecedented hardware integration to rapidly design and deploy measurement and control systems. The graphical language is named "G" abbreviated as Graphical Programming is a Dataflow type Programming Language where the programmer connects different function-nodes by drawing wires. LabVIEW is commonly used for data acquisition, instrument control, and industrial automation on a variety of platforms.

$D A Q$ Device (CFP2020): DAQ hardware acts as the interface between a computer and signals from the outside world. It primarily functions as a device that digitizes incoming analog signals so that a computer can interpret them [2]. The three key components of a DAQ device used for measuring a signal are the signal conditioning circuitry, analog-to-digital converter (ADC), and computer bus. Many DAQ devices include other functions for automating measurement systems and processes. For example, digital-toanalog converters (DACs) output analog signals, digital I/O lines input and output digital signals and counter/timers count and generate digital pulses.

Signal Conditioning: Signals from sensors or the outside world can be noisy or too dangerous to measure directly. Signal conditioning circuitry manipulates a signal into a form that is suitable for input into an ADC [3]. This circuitry can include amplification, attenuation, filtering, and isolation. Some DAQ devices include built-in signal conditioning designed for measuring specific types of sensors.

Driver software: Driver software provides application software the ability to interact with a DAQ device [9]. It simplifies communication with the DAQ device by abstracting low-level hardware commands and register-level programming. Typically, DAQ driver software exposes an ap- plication programming interface (API) that is used within a programming environment to build application software.

Computer Bus: DAQ devices connect to a computer through a slot or port. The computer bus serves as the communication interface between the DAQ device and computer for passing instructions and measured data. DAQ devices are offered on the most common computer buses including USB, PCI, PCI Express, and Ethernet. Ethernet communication is employed in this project.

\section{OFS AND HARDWARE SETUP}

Fiber optic weight sensors: A OFS (fiber optic sensor) is a sensor that uses optical fiber either as the sensing element ("intrinsic sensors"), or as a means of relaying signals from a remote sensor to the electronics that process the signals ("extrinsic sensors") shown in fig2. Fibers have many uses in remote sensing [3]. Depending on the application, fiber may be used because of its small size, or because no electrical power is needed at the remote location, or because many sensors can be multiplexed along the length of a fiber by using light wavelength shift for each sensor, or by sensing the time delay as light passes along the fiber through each sensor. Time delay can be determined using a device such as an optical time-domain reflects meter and wavelength shift can be calculated using an instrument implementing optical frequency domain reflectometry. Fiber Optic sensor system.
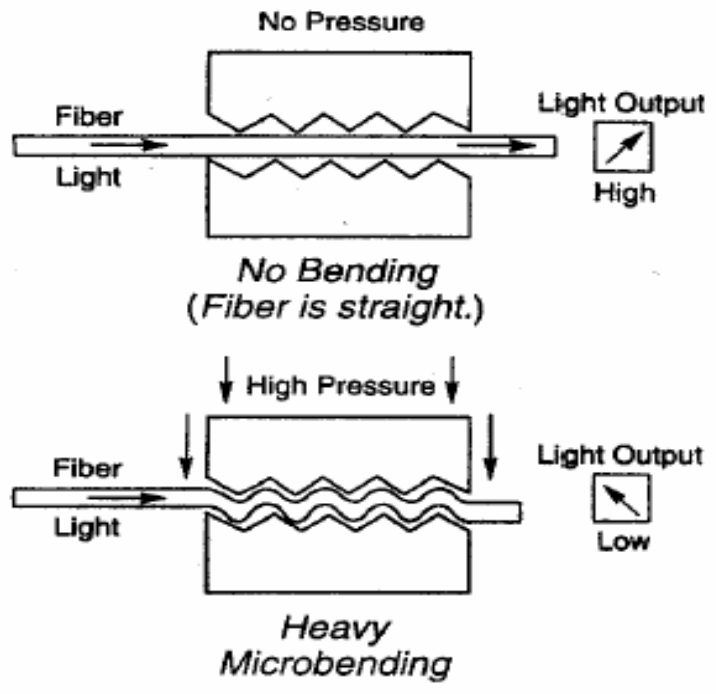

Figure 2. Microbend Sensor

SETUP: Cylindrical sticks of $1 \mathrm{~mm}$ of diameter are arranged in a pattern in order to create a corrugated pattern on both planes of the laid optical fiber is shown in fig.3. A LASER Source is incident continuously into the optical fiber as a simulation for the SSC at the other end of the fiber. An LDR circuit detects the amplitude of light passing through the fiber and then converts the non-electrical signal to an electrical signal. This voltage signal is given to the field point form where the VI takes the value for processing [4]. 

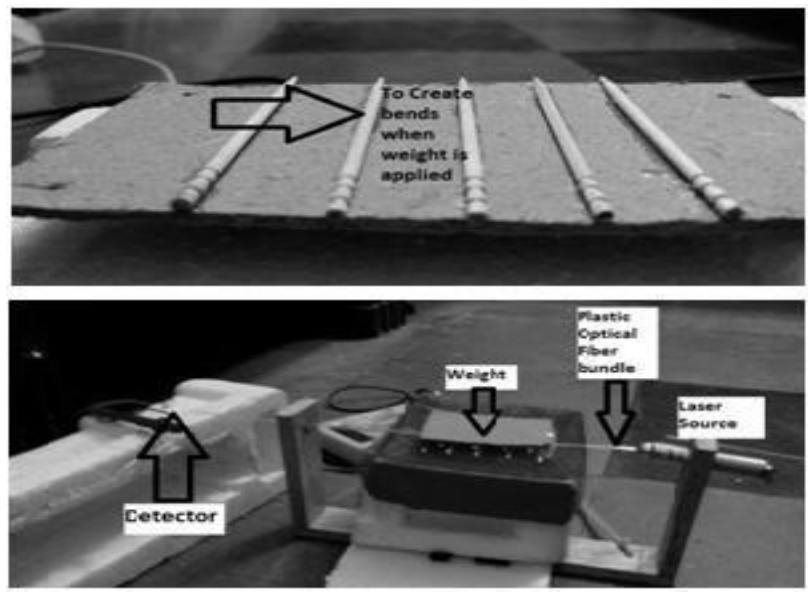

Figure.3. OFS sensor set up

Conveyor belt: A conveyor runs on two wheels fixed to shaft of 2 DC motors. The DC motors are used to drive the conveyor.

Motor driver- L293D: L293D is a dual H-bridge motor driver integrated circuit (IC) is shown in fig.4.Motor drivers act as current amplifiers since they take a low-current control signal and provide a higher-current signal. This higher current signal is used to drive the motors. L293D contains two inbuilt $\mathrm{H}$-bridge driver circuits. In its common mode of operation, two DC motors can be driven simultaneously, both in forward and reverse direction. The motor operations of two motors can be controlled by input logic at pins $2 \& 7$ and $10 \& 15$. Input logic 00 or 11 will stop the corresponding motor. Logic 01 and 10 will rotate it in clockwise and anticlockwise directions, respectively.

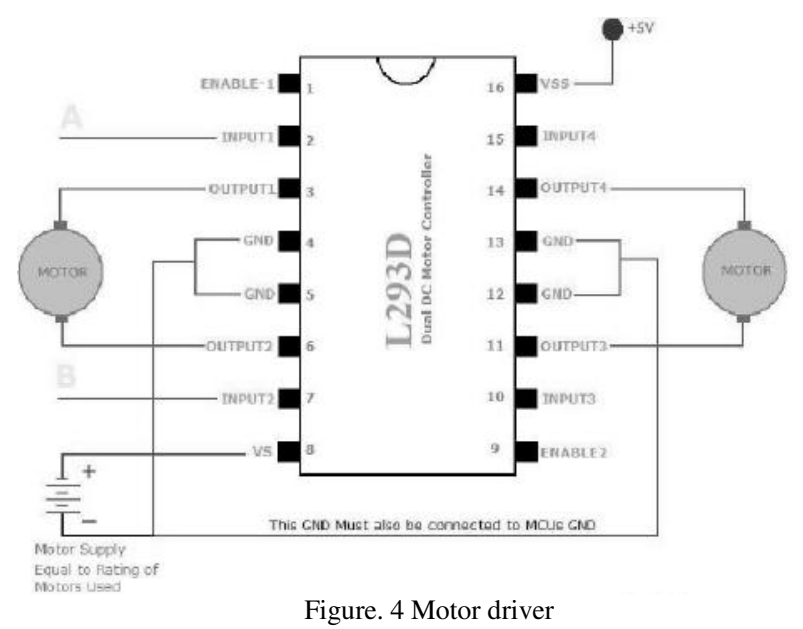

CFP2020: In this paper by Using the NI LabVIEW RealTime Module, powerful data-logging, control, and measurement systems on PC can be developed and application can be easily embedded on the NI CFP-2020 intelligent controller for reliable distributed or stand-alone deployment [9]. The cFP-2020 features an Ethernet port, four serial ports, removable Compact Flash storage, and extended DRAM memory for larger and more advanced applications. Engineers and scientists typically use cFP-20xx controllers in applications requiring industrial-grade reliability - such as stand-alone data-logging, analog process, and discrete con- trol systems - to run PID control loops, actuate valves and motors, take measurements, perform real-time analysis and simulation, log data, and communicate over serial, phone, and Ethernet.

CFP-AIO-610 (analog input output module): The National Instruments cFP-AIO-610 is an 8-channel, singleended, combination analog input/analog output module is shown in fig.5. It can measure four channels of voltage or $\mathrm{mA}$ current loops from industrial sensors and transmitters and can output four channels of 0 to 10 or $\pm 10 \mathrm{~V}$ to control valves, gauges, and other industrial actuators. The NI cFPAIO-610 has an internal update rate of $1.4 \mathrm{kHz}$, ideal for low-channel-count systems or PID control. The module includes over ranging and onboard diagnostics to ensure trouble-free installation and maintenance. The module also makes programming easier by automatically scaling and liberalizing input and output signals to avoid the step of converting binary numbers into engineering units in your control or monitoring software. The cFP-AIO-610 module is calibrated according to NIST-traceable calibration standards, ensuring accurate and reliable analog measurement and control.

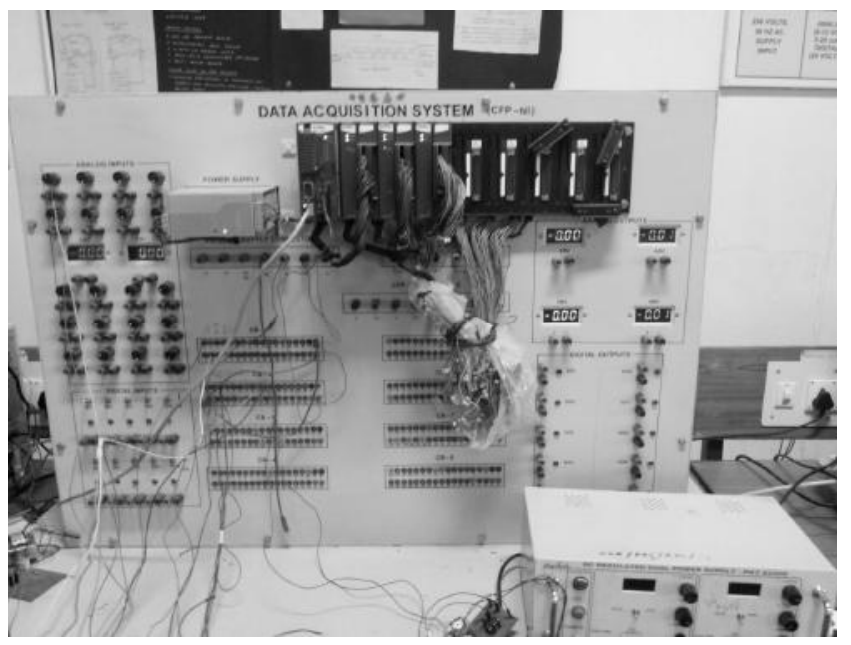

Figure.5.NI compact field point

The CFP-AIO-610 is a Compact Field Point analog input and output module with the following features:

1. Four analog voltage or current input channels with 11 input ranges up to $\pm 30 \mathrm{~V}$ or $\pm 20 \mathrm{~mA}( \pm 36 \mathrm{~V}$ or $\pm 24 \mathrm{~mA}$ with $20 \%$ over ranging)

2. Four analog voltage output channels with \pm 10 or 0 $10 \mathrm{~V}$ ranges $( \pm 10.2$ or $0-10.2 \mathrm{~V}$ with $2 \%$ over ranging)

3. $\quad 1.4 \mathrm{kHz}$ hardware update rate

4. 12-bit resolution

5. -40 to $70{ }^{\circ} \mathrm{C}$ operation

6. Voltage outputs sink or source up to $10 \mathrm{~mA}$ per channel

7. On-board diagnostics including one out-of-range indicator for all inputs and one over current indicator for each output.

8. Current inputs protected up to $\pm 10 \mathrm{~V}$. 
Wiring CFP-AI-100: Table 1 lists the terminal assignments for the signals associated with each channel. Each channel has separate input terminals for voltage (Vin) and current (Iin) input. Voltage and current inputs are referenced to the COM terminals. If an external supply is used to power field devices, power supply should be connected to the $\mathrm{V}$ and $\mathrm{C}$ terminals of the terminal base or connector block. The cFP-Al-100 has eight single-ended input channels. All eight channels share a common ground reference that is isolated from other modules in the Field Point system. Figure 5.8 shows the analog input circuitry on one channel

TABLE I

TERMINAL ASSIGNMENTS

\begin{tabular}{|c|c|c|c|c|}
\hline \multirow{2}{*}{ Channel } & \multicolumn{4}{|c|}{ Terminal Numbers } \\
\cline { 2 - 5 } & $\mathrm{V}_{\text {in }}$ & $\mathrm{I}_{\text {in }}$ & $\mathrm{V}_{\text {sup }}$ & COM \\
\hline \hline 0 & 1 & 2 & 17 & 18 \\
\hline 1 & 3 & 4 & 19 & 20 \\
\hline 2 & 5 & 6 & 21 & 22 \\
\hline 3 & 7 & 8 & 23 & 24 \\
\hline 4 & 9 & 10 & 25 & 26 \\
\hline 5 & 11 & 12 & 27 & 28 \\
\hline 6 & 13 & 14 & 29 & 30 \\
\hline 7 & 15 & 16 & 31 & 32 \\
\hline
\end{tabular}

pH sensor- $p H 614: \mathrm{pH}$ is the degree of acidity or alkalinity of a solution. $\mathrm{pH}$ measurement is very essential and crucial in any process plants where ever fluids are involved is shown in Fig.6. This inevitability is due to the metal fluid interaction during the processes [8]. Fluids involving process with boilers, pipelines, valves, stirrers, turbines, etc. need to be monitored for $\mathrm{pH}$. In Fluid filling plants like food processing, pharmaceutical, cosmetic, chemical, fertilizer, etc. need to be checked for $\mathrm{pH}$ at the process end as part of quality testing.

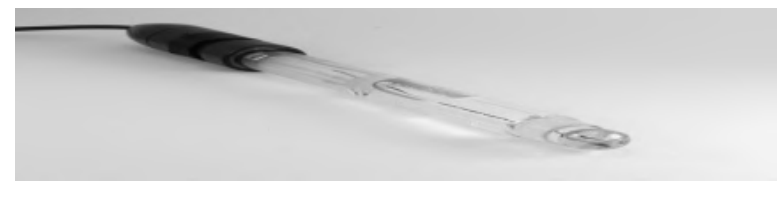

Figure.6. pH Sensor electrode

LDR as proximity sensor: An LDR acts as a proximity sensor. The LDR is given a continuous 5V DC power supply with signal conditioning is shown in fig.7. The light from ambience falls on the LDR and keeps the circuit ON [8]. As and when a filled bottle passes between the pair, interrupting the ambient light, the circuit breaks and the count in the LabVIEW VI increments by one. The count form this sensor is used as a base count for other sensor's output analyzing to determine efficiency.

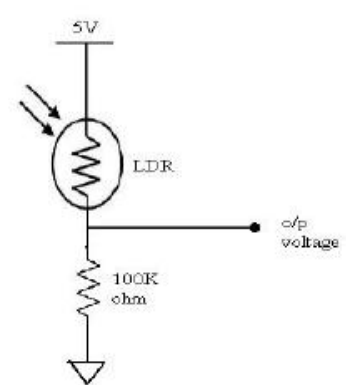

Figure.7. LDR Signal Conditioning

NI CFPTC 120: The National Instruments cFP-TC-120 is an 8-channel input module for direct measurement of temperature from standard J, K, T, N, R, S, E, and B thermocouple(Fig.8) types. With signal conditioning, doubleinsulated isolation, input noise filtering, and a high-accuracy delta-sigma 16-bit analog-to-digital converter, the NI cFPTC-120 delivers reliable, accurate temperature or mill volt measurements. Designed from top to bottom for productivity and reliability, the cFP-TC-120 features Hot PnP (plugand-play) operation, easy configuration, self-diagnostics, and automatic scaling to engineering units [7].

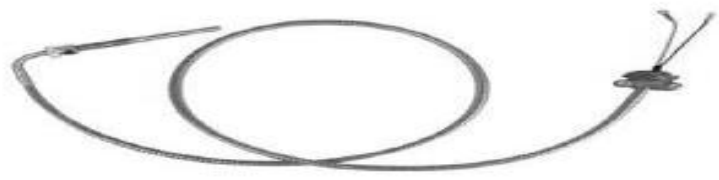

Figure.8 Thermocouple

\section{LABVIEW INTERFACING AND VI CODE}

This section deals with interfacing and locating- pins and icons for VI development is shown in (Fig.9 and Fig.11). It contains details to develop a VI and insight to various LabVIEW functions used. LabVIEW follows a dataflow model for running VIs. A block diagram node executes when it receives all required inputs. When a node executes, it produces output data and passes the data to the next node in the dataflow path. The movement of data through the nodes determines the execution order of the VIs and functions on the block diagram.

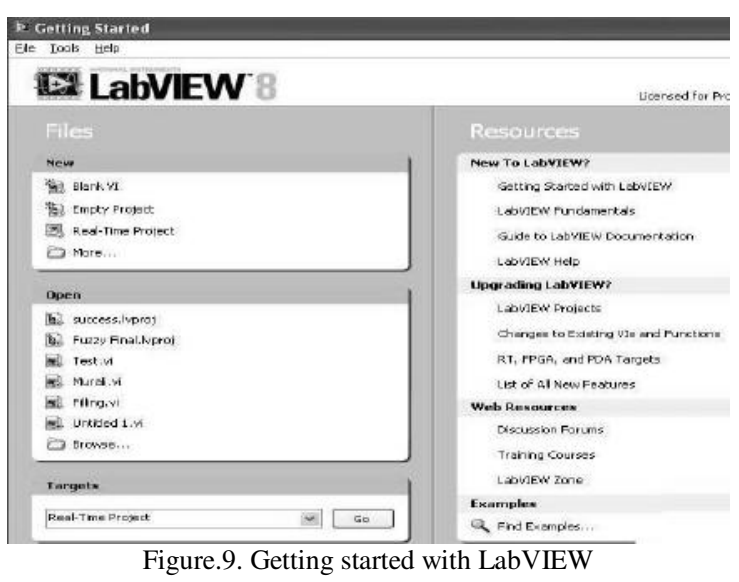




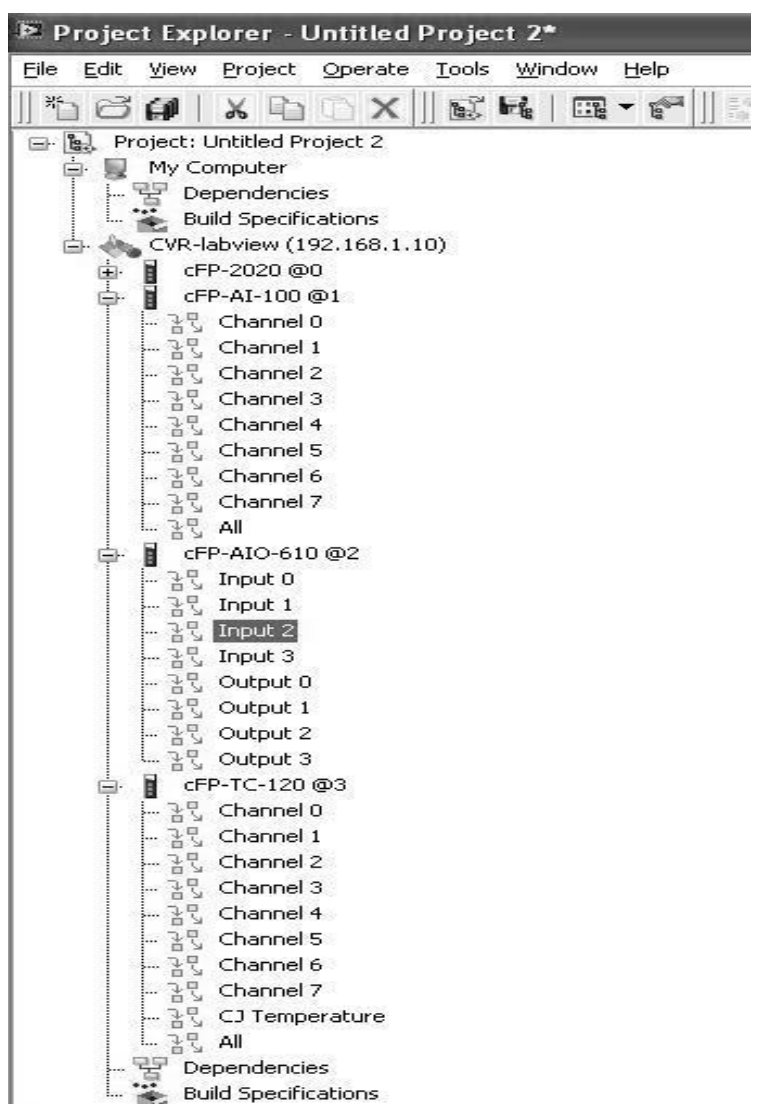

Figure. 10.Channels of NI modules

LabVIEW (Laboratory Virtual Instrumentation Engineering Workbench) is a highly productive development environment that engineers and scientists use for graphical programming and unprecedented hardware integration to rapidly design and deploy measurement and control systems. The graphical language is named "G" abbreviated as Graphical Programming is a Dataflow type Programming Language where the programmer connects different function-nodes by drawing wires. LabVIEW is commonly used for data acquisition, instrument control, and industrial automation on a variety of platforms.

Virtual Instrumentation is the use of customizable software and modular measurement hardware to create userdefined measurement systems, called virtual instruments Traditional' or 'natural' instrumentation systems are made up of pre-defined hardware components, such as digital multimeters and oscilloscopes that are completely specific to their stimulus, analysis, or measurement function. Because of their hard-coded function, these systems are more limited in their versatility than virtual instrumentation systems. The primary difference between 'natural' instrumentation and virtual instrumentation is the software component of a virtual instrument.

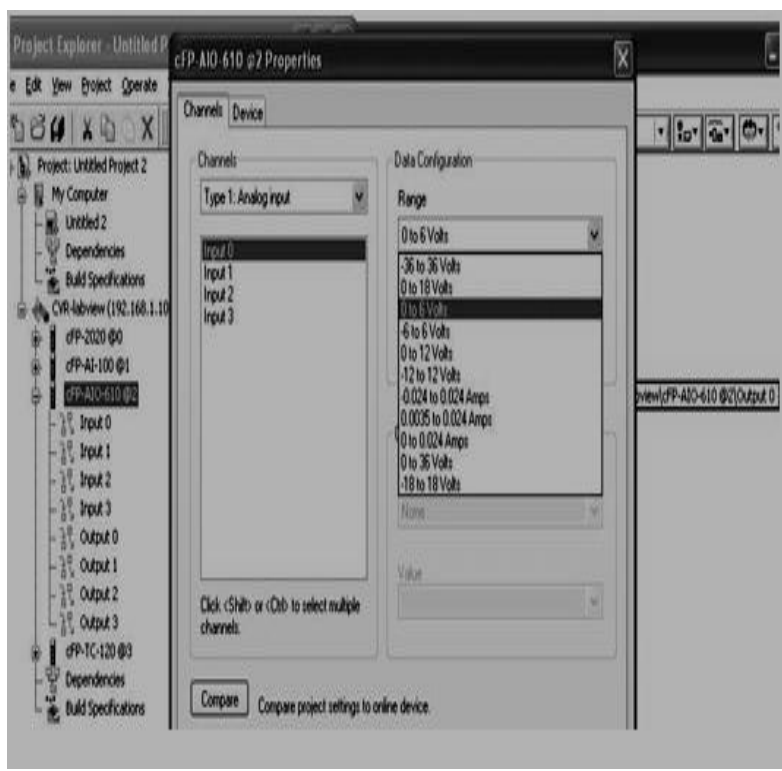

Figure. 11. Data configuration of pins

\section{Working of the project}

1. Empty bottles are placed on the conveyor.

2. When the empty bottle comes under the overhead tank for filling, LDR sensor detects the bottle.

3. The actuator stops the conveyor belt.

4. The Solenoid valve opens and the water from the overhead tank starts filling the bottle.

5. After the bottle is full (after a time delay) CFP signal changes and the bottle starts moving again.

6. In parallel, the tank is monitored for level, $\mathrm{Ph}$ and temperature.

7. Then, the bottle moves to the optical fiber strain gauge where the weight is measured and is logged to the spread sheet file with a time stamp.

\section{RESULTS}

Results observed: The optical fiber sensor designed works linearly in the given range. The entire process has been automated and the quality test of the end product has been done. The data from the sensors and measuring devices has been logged by the VI and stored in various formats. The overhead tank level has been controlled by ON OFF control algorithm. Various process parameters like $\mathrm{pH}$, temperature and other process parameters have been monitored. The result can be observed from fig. 12 to fig. 15 .

Scope of the project: The scope of applying the sensors and controlling process used in this project is wide and huge. In the present project, the data acquisition is done using Ethernet protocol and communication. There is scope in LabVIEW that the VI can be used to control industrial field bus in controlling, monitoring and modifying through internet communication. In many industries like pharmaceutical, cosmetics, food processing, fertilizer, chemical, these processes are of inevitable use. 


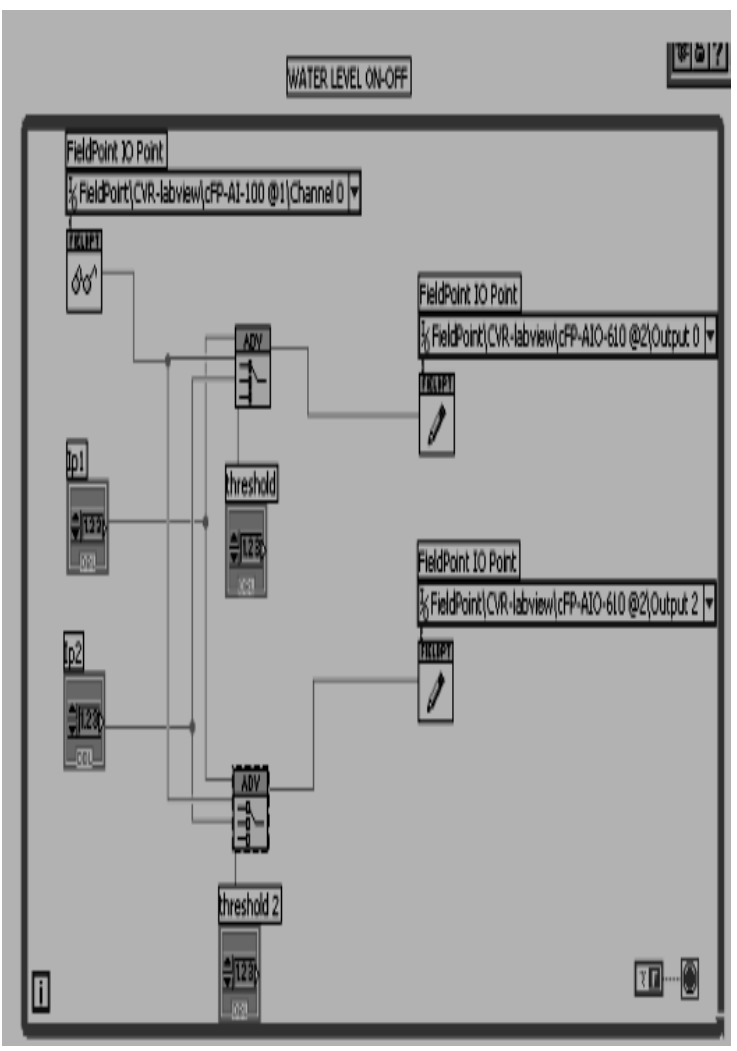

Figure.12. Water Level ON-OFF

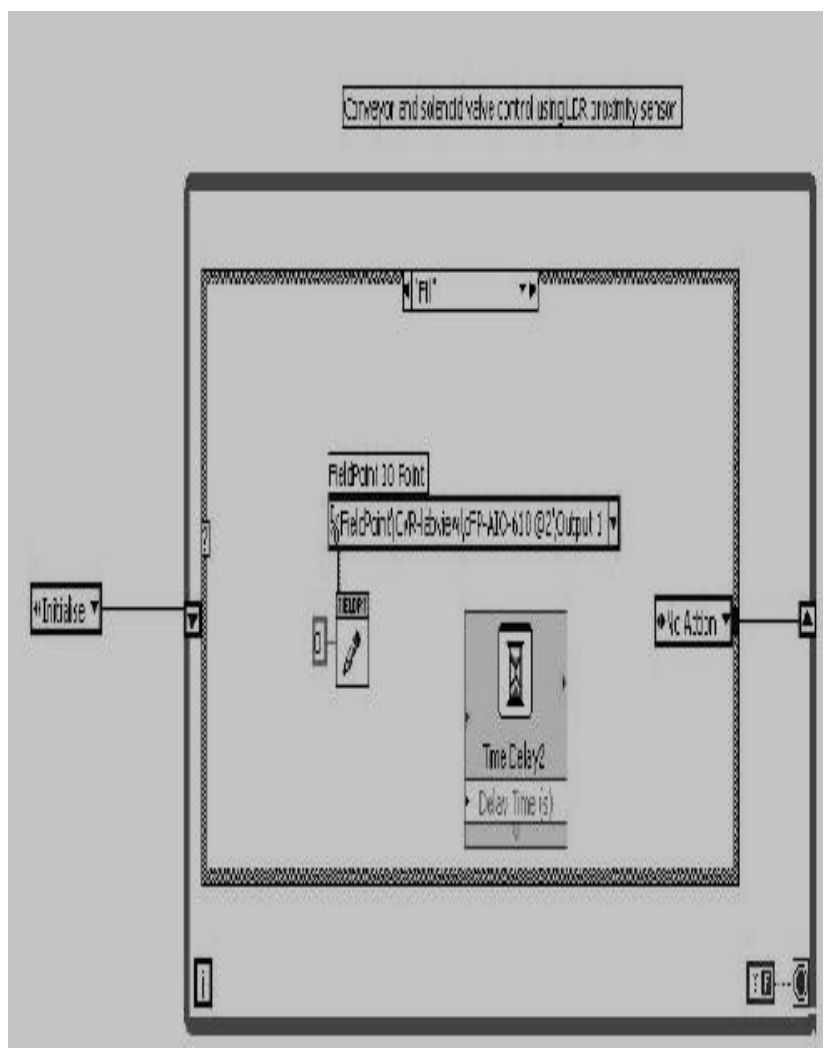

Figure.13. Fill case of case structure

QUALLITY TESTING

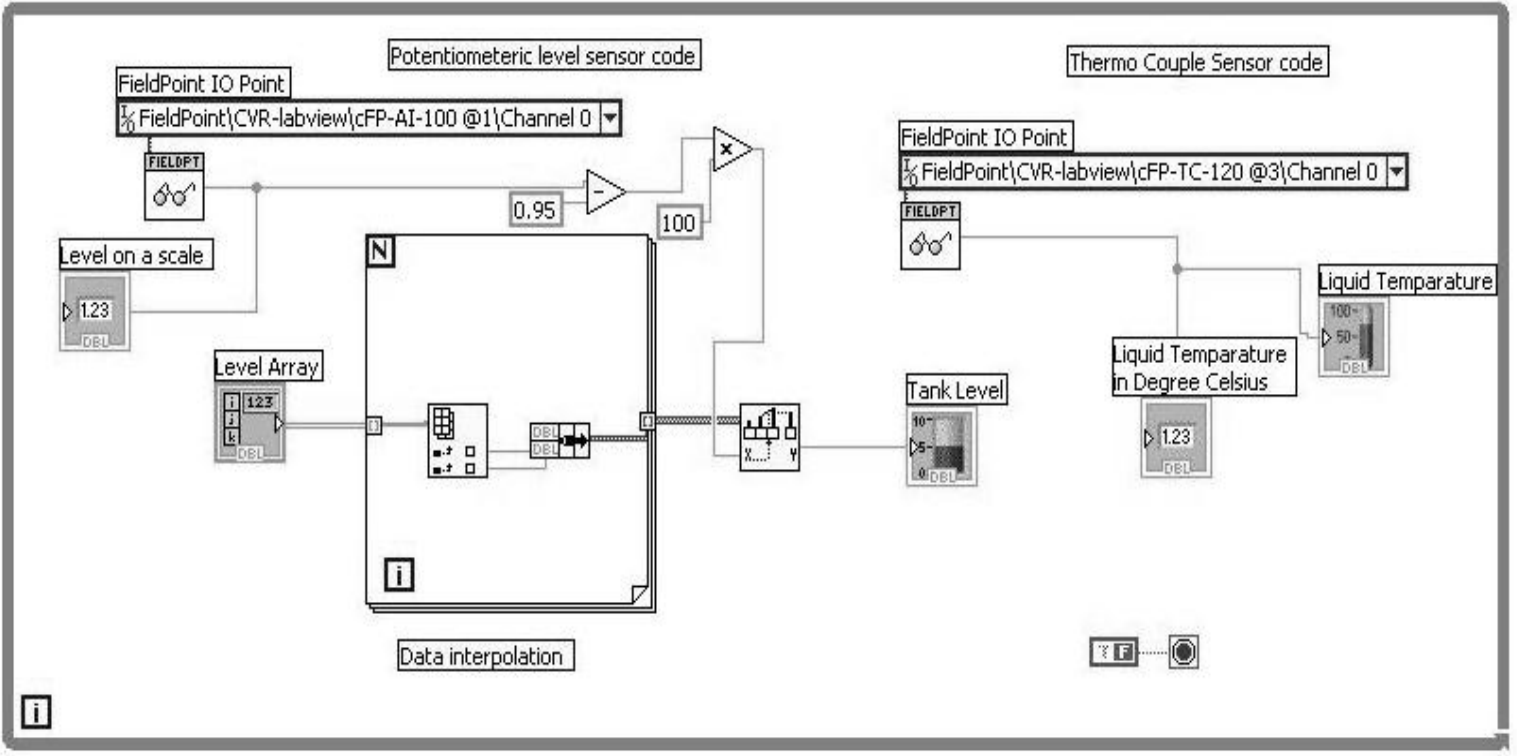

Figure.14.Blockdiagram of the application 


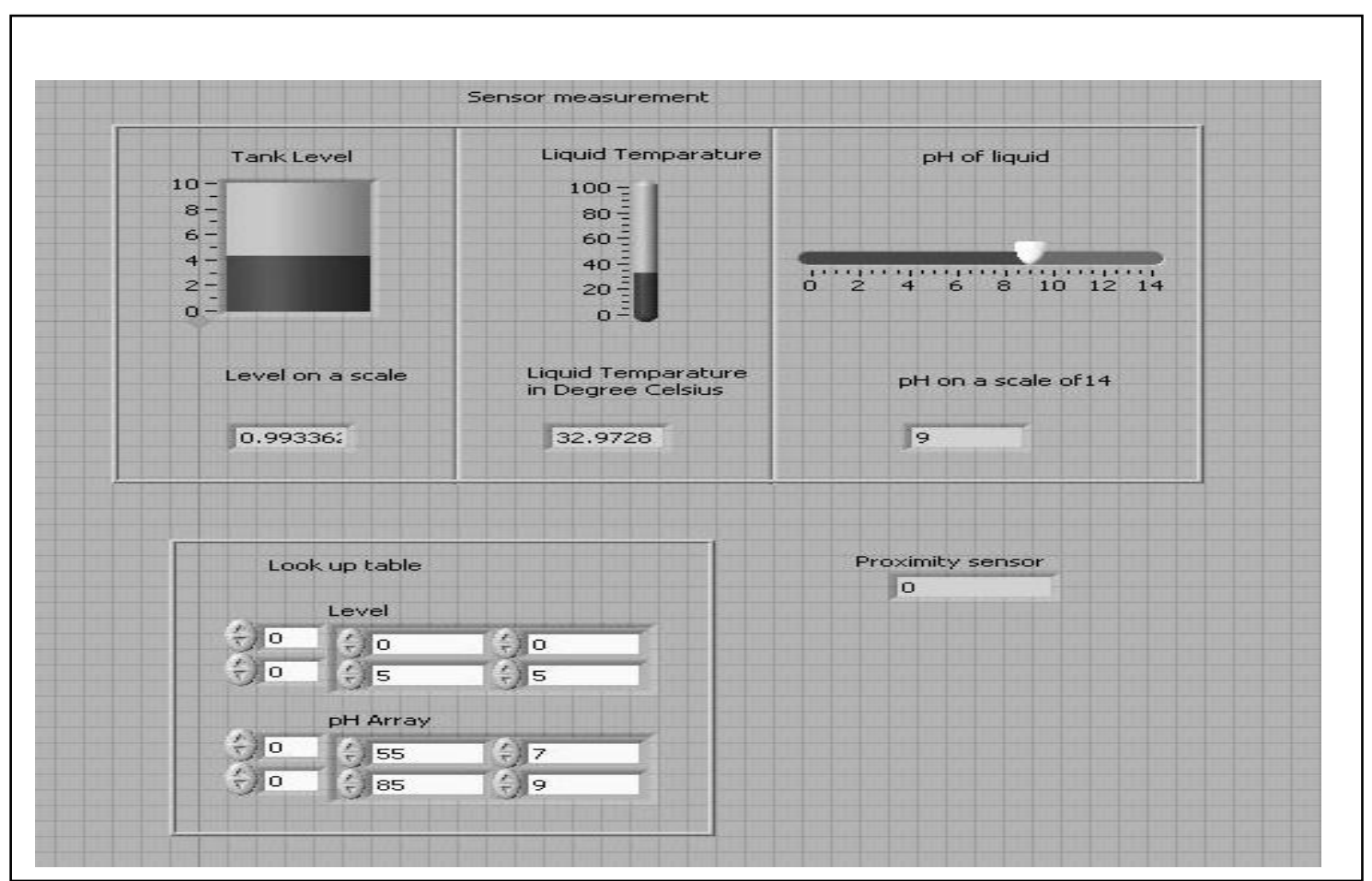

Figure.15.Front panel of the application

\section{CONCLUSIONS}

In this paper, the automatic bottle filling plant is implemented. The sensor applications are studied and operated to fully understand their characteristics and operation. This operational experience is used to implement the sensors appropriately. The automation, controlling, monitoring, data processing, of the bottle filling plant has been done by a Virtual Instrument developed on LabVIEW platform.

\section{REFERENCES}

[1]. Efendioglu HS, Yildirim T, Fidanboylu K,'Prediction of Force Measurements of a Micro bend Sensor Based on an Artificial Neural Network" 9 September 2009, the Special Issue Neural Networks and Sensors ,ISSN 1424-8220.

[2]. Kevin James "PC Interfacing and Data Acquisition Techniques for Measurement, Instrumentation and Control", 1st edition. Newnes, 17-Jul-2000 Pages 50-70.

[3]. Senior John M, "Optical Fiber Communications" Pearson Education India, 01-Sep-2009, Pages 90-120.

[4]. Liang Feng, Ye-Long Xu, William S Fegadolli, Ming-Hui Lu, José EB Oliveira, "Experimental demonstration of a unidirectional reflectionless parity-time metamaterial at optical frequencies".Journal:Nature-materials, Volume-12,Issue 2,Pages:108-113.

[5]. Ronald L.Krutz,"Securing SCADA system". Wiley, 28-Nov2005 Pages: 40-50.

[6]. Sabeetha Begum.M and Kumarnath.J "LABVIEW Based Module For Bio Signals Monitoring", IJCSMC, Vol. 3, Issue. 2, February 2014, pg.423-431.

[7]. Jeffrey Travis "LabVIEW for Everyone (National Instruments virtual instrumentation)"second edition, 16 Nov 2001 pg.4255 .
[8]. A.K. Sawhney, Puneet Sawhney "A course in Electrical and Electronic Measurements and Instrumentation",Dhanpat Rai Publications,pg.56-78 \& 450-465.

[9]. http://www.ni.com/labview/. 